\title{
Die Herausforderung durch die Anderen 500 Jahre Christentum in Lateinamerika: Conquista, Sklaverei, Befreiung
}

von Paulo Suess

\section{Die Armen und die Anderen}

Der Hunger der Armen und die Legitimationspflicht des Anderen sind gleichermaßen ein Skandal, der das Leben von Völkern, sozialen Gruppen und Individuen bedroht. Die weltweite Armut, Ergebnis einer historisch-strukturellen, sozio-ökonomischen Asymmetrie, muß solidarisch bekämpft werden. Das Anderssein jedoch - jene identitätsbildende Widerstandskraft gegen administrative, technokratische und neokoloniale Gleichmacherei - ist ein grundsätzlich verteidigenswertes Gut. Partizipative, demokratische Gleichheit und universale Solidarität haben die Überwindung von Armut und die Anerkennung der Anderen - andere Ethnien, Kulturen, Glaubensbekenntnisse - im Auge. Das Anderssein der Anderen hat nur dann kein Recht auf Anerkennung, wenn es Hegemonieansprüche anmeldet, das Gespräch verweigert und den Toleranzvertrag, der ja die Voraussetzung für modernes gesellschaftliches Zusammenleben bildet, grundsätzlich nicht anerkennt.

Sozialanalytiker geben zu bedenken, daß die Anerkennung einer unendlichen Vielzahl von verschiedenen Lebenswelten ein vom bösen (Klassen-) Feind ins Feld gestreuter Spaltpilz sein könnte, der das universale Befreiungsprojekt der Ausgebeuteten nicht nur verzögere, sondern häufig boykottiere. Kulturelles Anderssein könne die Wurzel sozio-ökonomischer Ausbeutung sein und müsse daher immer auch unter Ideologieverdacht gestellt werden. Schließlich behaupten sie, Indios und die Nachfahren der Negersklaven in Lateinamerika seien eben Arme.Um dann aber doch noch irgendeine Differenzierung anzudeuten, wurde die Rede von den „Ärmsten der Armen“ eingeführt. Unter dem Paradigma „Ärmste der Armen“ wird dann die Sache der Indios, der Nachfahren der afro-amerikanischen Sklaverei, manchmal auch die der Frauen und anderer vom nationalstaatlichen. Standard abweichender kultureller Minderheiten verhandelt.

Mit der Reduktion des Anderen zum Armen aber wird gerade jener Pfeil gebrochen, mit dem die Unterdrückten Lateinamerikas 500 Jahre lang Wider-

Prof. Dr. Paulo Suess war Beauftragter der brasilianischen Bischofskonferenz für Indianerfragen und bis 1986 Generalsekretär des CiMi (Conselho indigenista missionaria) der kath. Kirche; z. Zt. arbeitet er als Missionswissenschaftler im Postgraduiertenkolleg der kirchlichen Hochschule FAI in São Paulo. 
stand geleistet haben. Überlebensstrategien in Lateinamerika gingen nicht von der durch Armut erzeugten Solidaritätsgemeinschaft, sondern von der Widerstandskraft der in den Untergrund verbannten Kulturen aus. In jedem Armen lebt ganz konkret ein Anderer: ein Guarani, ein Aymara, ein Landloser aus Amazonien, ein Afroamerikaner aus Bahia, ein Mestize der Andenregion. Kultur ist nicht das Privileg autochtoner Völker. Auch die Slumbewohner und die Bettlergruppe, die unter der Straßenüberführung wohnt, sind Subjekt von Kultur. Die wahre Identität lateinamerikanischer sozialer Gruppen und Völker muß unter der Decke fiktiver Nationalkulturen und gleichmacherischem Elend gesucht werden.

Andere sind nicht nur all jene, die von der Integration in die summarisch verordnete Identität der Nationalstaaten bedroht sind. Andere in der Dritten Welt sind heute mehr und mehr jene "ganz Anderen“, die nicht einmal mehr ausgebeutet werden, weil sie außerhalb der Prozesse von Mehrwertsaneignung durch Arbeit und Markt leben.

Die Realität sozialer Gruppen und Staaten Lateinamerikas hat also zwei strukturell ganz verschiedene Seiten: die soziale Monotonie von Hunger und Elend und die kulturelle Vielfalt des Andersseins. Ein Evangelisationsprojekt wird nur dann alternativ sein können, wenn es Perspektiven aufzeigt, welche die Monotonie von Hunger und Elend durch vielfältige Weisen von Solidarität und Gerechtigkeit unterbrechen. Die Utopie der Hungernden ist nicht die Monotonie des vollen Bauchs. Auch Gerechtigkeit, internationales Recht und Solidarität sind kulturell eingefärbt. Eine in der Perspektive der Nachfolge gelebte Option für die Armen muß die plurikulturelle Dimension im Auge behalten, die das Paradigma „Inkulturation“ einklagt. Diese Nachfolge als Option für die Armen führt mehr und mehr aus den formal-administrativen, normativ vereinheitlichten Sphären von Staat und Kirche heraus in jene Frei- und Untergrundzonen „informell-diversifizierter Ökonomie“, welche die etablierten Heilsangebote unterlaufen.

Eine Grundfrage lateinamerikanischer Kirchengeschichte, ob es überhaupt Evangelisierung oder eben nur Religionswechsel aus strategischen Gründen, eben nur Christianisierung, gegeben habe, ist nur am Anderen und nicht am Armen festzumachen. Brot hat keine Religion. Auch Heiden nähren sich und andere redlich. Kirchenbeschlüsse zeigen - nicht nur in diesen fünf Jahrhunderten seit der Conquista Amerikas - in der Regel eine barmherzige Akzeptanz des Armen und eine irritierte Intoleranz gegenüber dem Anderen. Bei den Armen in der Kirche aber hat es sich nie um schlichtweg Arme, sondern um „arme Christen", also um integrierte Andere, gehandelt.

Erst wo mit der Armenfürsorge auch das weiter gefaßte Kommunikationsund Identitätsproblem des Anderen akzeptiert wird, findet ein qualitativer Sprung zu integraler Befreiung statt. Von Evangelisation kann füglich erst dann die Rede sein, wenn integrale und universale Befreiung im Horizont lokaler Verständigung buchstabiert wird. Die historische Gretchenfrage an das Christentum lautet daher: „Wie hälst du es mit dem Anderen?“ 


\section{Artikulation}

Das Jahr 1492 war nicht nur das Jahr der sog. Entdeckung Amerikas. 1492 war auch - nach 900jähriger Präsenz des Islam auf der iberischen Halbinsel das Jahr der Unterwerfung der Mauren Granadas durch Spanien. 1492 war schließlich das Jahr, in dem die katholischen Könige spanische Juden vor die Alternative zwischen Zwangstaufe und Exil gestellt haben. In der Einleitung zum Bordbuch seiner ersten Amerikareise bringt Kolumbus Maurenunterwerfung, Judenvertreibung und Indioconquista miteinander in Beziehung: „Im gegenwärtigen Jahr 1492, nachdem eure Hoheiten den Krieg gegen die Mauren (...) beendet hatten (...), sah ich, wie die königlichen Fahnen Eurer Hoheiten an den Türmen der Alhambrafestung gehißt wurden (...). Nachdem Eure Hoheiten alle Juden aus all Ihren Herrschaftsbereichen vertrieben hatten, sollte ich (...) mich in die genannten Gegenden nach Indien aufmachen (...).“

Doch Kolumbus, der Glaubensbote, war auch gleichzeitig Goldsucher, Lehensherr und Sklavenfänger. Schon im Tagebuch seiner ersten Amerikareise ist $77 \mathrm{mal}$ von Gold die Rede. Bartolomé und Diego Kolumbus, zwei Brüder des Entdeckeradmirals Christoph, beginnen bald mit dem Export von Indiosklaven nach Spanien. ${ }^{2}$ Kolumbus selbst schlägt vor, den Indiosklavenhandel mit der Metropole zu legalisieren, „bis dieses Geschäft auf die Beine kommt", das ,jetzt sehr verloren ist, weil diese Leute nichts taugen und die Indios keinen Tribut zahlen".3

In der Instruktion vom 16. September 1501 an Nicolás de Ovando, dem neuen Gouverneur Westindiens, erinnern die katholischen Könige daran, „daß die Indios Uns unsere Tribute und Rechte zahlen (..); alles geschehe so, wie es zum Nutzen und Wohl unseres Einkommens ist" ${ }^{4}{ }^{4}$ Und nachdem Königin Isabel die Berichte Ovandos erhalten hatte, ordnete sie am 20. Dezember 1503 an, daß

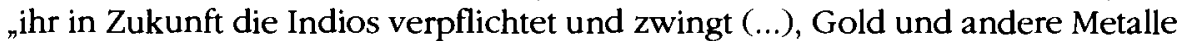
herbeizuschaffen und landwirtschaftliche Produkte und Lebensmittel für die Christen zu besorgen (...)."

Der Königliche Erlaß von Medina legalisiert die Repartimientos - "jene tyrannische Pest (...) des Repartimiento, die später Ecomienda genannt wurde“ 6 - und definiert ihre Bedingungen: befristete Zwangsarbeit für einen vom Arbeitgeber festgelegten Lohn. Der ständige „Arbeitgeberwechsel“ der zur Zwangsarbeit abkommandierten Indios ermöglichte ihre extreme Ausbeutung. Daher begannen die Ordensleute allmählich die lebenslängliche Arbeitszuweisung an einen bestimmten Herrn für das geringere Übel zu halten, weil sie dabei auf eine „rationalere" Ausbeutung der indianischen Arbeitskraft hofften. Auch der Import von Negersklaven wurde seit 1505 als komplementäre Maßnahme für den gespannten Arbeitsmarkt ins Auge gefaßt. ${ }^{7}$ Im gleichen Jahr erbat König Fernando vom Papst die Patronatsrechte über die Missionskirche, die ihm schließlich am 28. Juli 1508 zugestanden wurden. ${ }^{8}$

In Westindien wiederholt Spanien, was ihm 750 Jahre vorher selbst widerfahren war. In der Mozarabischen Chronik erscheint das "unglückliche" und "verurteilte“ Spanien entvölkert durch das Schwert,den Hunger und die Sklaverei. Seine Städte sind den Flammen übergeben. Die Menschen sterben gekreu- 
zigt, enthauptet oder fliehen in die Wälder. Spanien Unglück ist nur dem Trojas, Jerusalems, Babyloniens oder Roms vergleichbar. ${ }^{9}$ Mit der Conquista Amerikas wiederholt sich die spanische Tragödie in Amerika. Las Casas erinnert sich in einem theologisch sehr dichten Text seiner 'Historia de las Indias' der Ureinwohner Westindiens: „Ich habe in Westindien Jesus Christus, unseren Heiland, zurückgelassen, nicht einmal, sondern tausend Male, gegeißelt, gequält, geohrfeigt und gekreuzigt durch die Spanier, die diese Leute heimsuchen und zugrunde richten. Während sie den Indios vorzeitig das Leben nehmen, nehmen sie ihnen auch die Möglichkeit ihrer Bekehrung und Buße. So sterben sie ohne Glaube und Sakramente. "10

Aber auch Las Casas, der als Bischof von Chiapas (Mexiko), Spaniern, die Indios auf ihren Encomiendas ausbeuteten, die Absolution im Beichtstuhl verweigerte, hat die Negersklaverei lange Zeit nicht grundsätzlich in Frage gestellt. In einer Denkschrift aus dem Jahre 1516 bittet er von Kardinal Cisneros „20 Neger und andere Sklaven" für jedes Dorf seines Kolonisationsprojekts, um die ausgebeuteten Indios zu ersetzen. ${ }^{11}$ Der ersten Bittschrift um Negersklaven folgen andere. ${ }^{12}$ Noch im Jahre 1543 , jetzt schon zum Bischof ernannt, bittet er Karl V. um 24 Negersklaven. ${ }^{13}$ Am 13. Februar 1544 erhält Las Casas schließlich die Erlaubnis, vier Sklaven zu seinen persönlichen Diensten und ohne "Lagerkosten" in seine Diözese mitzunehmen ${ }^{14}$ Erst viele Jahre später, bei der Abfassung seiner ,Historia de las Indias', jetzt schon aus Amerika endgültig nach Spanien zurückgekehrt, erklärt sich Las Casas „schuldig aus Unwissenheit“, hatte er doch geglaubt, daß es sich bei den Negern um "rechtmäßig versklavte“ Afrikaner gehandelt habe; er bekennt: „die Sklaverei der Neger war so ungerecht wie die der Indios". ${ }^{15}$

Die Sache der Unterdrückten und Ausgeschlossenen darf nicht durch eine interne Hierarchisierung halbiert werden, mag es sich da um Mauren, Juden, Indios oder Negersklaven handeln. Binnensolidarität ist notwendigerweise zum Scheitern verurteilt. Erst die Artikulation sozialer Ausbeutung mit kultureller Marginalisierung und religiöser Diskriminierung eröffnet einen je spezifischen, universalen Befreiungshorizont.

Auch heute kann die Sache der Indios nicht wirksam verteidigt werden, wenn sie nicht gleichzeitig im Kontext globaler Befreiung vorangetrieben wird. Die Sache der Indios - Befreiung von Integrationsdruck, Selbstbestimmung auf gemeinschaftlich verwaltetem Land, Bodensicherung, pfleglicher Umgang mit der Natur, Autonomie kultureller Nationalität - ist nicht isoliert mehrheitsfähig. Das war der eigentliche Grund, warum die Jesuitenreduktionen in Paraguay gescheitert sind. Im Meer kolonialer Ausbeutung gibt es heute keine Überlebensaussichten auf Inseln einer "heilen" Welt. Daher muß Solidarität mit indianischen Völkern heute aufzeigen, daß sie gesamtmenschheitliche Überlebensinteressen zur Sprache bringt. Die Sache der Indios ist auch die Sache der Landund Obdachlosen; die Sache Amazoniens; die Sache der von Todesschwadronen verfolgten Straßenkinder; die Sache der Arbeitslosen und der politischen Flüchtlinge. Europäische Solidarität mit den fernen Yanomami beginnt bei den Türken in Berlin, bei den Roma und Sinti, bei all denen, die im europäischen Nahbereich Ausgrenzungsversuchen oder Integrationszwängen ausgesetzt sind. 


\section{Ambivalenz}

Für die Gewalttätigkeiten der Conquista kann nicht einfach der spanische oder portugiesische Nationalcharakter verantwortlich gemacht werden. Wer den Wurzeln vieler Gewalttätigkeiten in der Geschichte nachgeht, wird feststellen, daß Ursache und Legitimation dieser Gewalttätigkeiten oft auf absolute Wahrheitsansprüche zurückgehen. Ohne "Gott in Spanien" hätte es - im 16. Jahrhundert jedenfalls - auch kein Gold in Westindien gegeben. Die Conquista wirft die Frage nach der Ambivalenz des Christentums überhaupt auf. Obwohl die Zerstörung von Leben - 'den Indios vorzeitig das Leben nehmen', nannte es Las Casas - und die Kolonisierung von Völkern im Evangelium keine Argumente finden, waren es Christen, die im Namen des Evangeliums jüdische Synagogen zerstörten, heidnische Tempel niederbrannten, Häretiker an den Galgen brachten. Seit Konstantin bildete das Anathema des Anderen gleichsam die Oberstimme zu den Seligpreisungen des Gleichen.

Man braucht da nur die Judenpredigten bei den Kirchenvätern, bei Augustinus und Ambrosius etwa, nachzulesen. ${ }^{16}$ Im Jahre 388 verteidigt Ambrosius die Brandstifter der Synagoge von Kallinikón (Euphrat). Der Mailänder Bischof qualifiziert die Synagoge als „einen Ort der Ungläubigkeit, als die Heimat der Gottlosigkeit, als das von Gott selbst verurteilte Versteck des Wahnsinns" ${ }^{17}{ }^{17} \mathrm{Er}$ selbst, Ambrosius, würde die Synagogen Mailands zerstören lassen, wenn dies nicht schon vor langer Zeit geschehen wäre.

Und Bernhard von Clairvaux (gest. 1153) heißt in seinem Traktat „Über die Ehren der neuen Militz" die Soldaten Christi kämpfen "ohne die geringste Furcht zu sündigen, weil sie sich der Todesgefahr aussetzen oder den Feind töteten. Für sie bedeutet sterben oder töten für Christus kein Verbrechen, sondern bringt große Ehre mit sich“ ${ }^{18}$ Mit der Gründung der Militärorden wird auch die missionarische Sache mehr und mehr militarisiert. ${ }^{19}$

In der Bulle 'Romanus Pontifex', aus dem Jahre 1454, also 40 Jahre vor der Conquista Amerikas, gibt Papst Nikolaus V. dem portugiesischen Infanten Don Henrique „alle Rechte“, um in die Territorien der Sarazenen, Heiden oder sonstwelcher Feinde Christi „einzufallen, sie zu erobern, dort zu kämpfen, und diese zu besiegen und zu unterwerfen “. ${ }^{20}$ Der Infant könne die verdiente Todesstrafe dieser Feinde Christi in lebenslängliche Sklaverei verwandeln, könne ihre Güter konfiszieren und ihre Länder besetzt halten.

Conquista und Christianisierung Amerikas bedeuten nicht Bruch, sondern Kontinuität mit der nachkonstantinischen missionarischen Praxis. Die geschlossene Welt des Kirchenpatronats hat es ermöglicht, viele mittelalterliche Strukturen und Doktrinen in die lateinamerikanische Christenheit hinüberzuschaffen. Sie hielt diese von „reformerischen Umtrieben“ Andersgläubiger frei und ließ prophetische Stimmen nie zu strukturellen Störfaktoren ausarten. Die für Europa mit der Entdeckung Amerikas beginnende Neuzeit galt nicht für Amerika selbst. Leibeigenschaft der Indios im Feudalsystem der Kommenden und Sklaverei großen Umfangs, die ultramarine Plünderung der Edelmetalle, strengste Zensur von Lehr- und Geschichtsbüchern, Verfolgung und Vertreibung Andersgläubiger, Inquisition und Kampagnen zur Ausrottung der Idolatrie - das alles wies noch mehr ins Mittelalter als in die heraufziehende Neuzeit. 
Die Capitulatio de partibus Saxoniae (782) Karls des Großen z. B. ließ den Sachsen die gleiche Alternative wie das sog. Requerimiento (1513) den Indios: entweder Bekehrung zum Christianismus oder Tod. Wenn der Gründer des sog. christlichen Europa im Jahre 782 in Verden an einem einzigen Tag 4.500 „Aufständische" niedermetzeln ließ, so war dies nicht so etwas ganz anderes im Vergleich zu Cortés, der „zur Vorbeugung“ eine große Zahl Indios aus Churultecal in einen Saal einsperren und dann niedermetzeln ließ. „In wenigen Stunden“, so Cortés, „starben mehr als dreitausend Männer“."21

Aus dem heiligen Jakobus, dem im Befreiungskampf gegen die muslimischen Mauren angerufenen „Maurentöter", wurde in Amerika der Heilige der Unterwerfungsfeldzüge gegen die Indios. Zahlreiche Legenden berichten, wie Jakobus, der Indiotöter (Santiago, o Mataindios), bei vielen aussichtslosen Schlachten auf einem weißen Pferd wie ein apokalyptischer Reiter erschienen sei und die Kriegswende für die Spanier herbeigeführt habe ${ }^{22}$ Die zahlreichen "Santiago" geweihten Städte Spanisch-Amerikas sind Zeugen solcher Angriffskriege, die mit der religiösen Rückendeckung des spanischen Nationalheiligen geführt wurden. Das Bündnis zwischen Kreuz und Schwert spielte eine entscheidende Rolle bei der Gründung des christlichen Europa wie bei der Schmiede des katholischen Amerika.

Die Konquistadoren auf dem Weg nach Mexiko waren überrascht, als sie die große Zahl indianischer Heiligtümer sahen, ,voller Dämonen und teuflischer Figuren“. Bernal Diaz, Soldat und Berichterstatter der Conquista Mexikos, beschreibt die Zerstörung der Götterstatuen der Totonacos, „die abschreckenden Drachen gleichten, so groß wie Kälber (...). Und als die Kaziken und Priester, die bei ihnen waren, sie so in Scherben zerschlagen sahen, da weinten sie und bedeckten die Augen und in ihrer Totonakensprache baten sie ihre Götter um Vergebung, dies alles sei ja nicht mehr in ihrer Hand und die Verantwortung dafür sei nicht ihnen anzurechnen, wohl aber diesen Weißen, die sie zerstört hätten (...).'23

Daraufhin befahl Cortés den Priestern, die Scherben zusammenzutragen und zu verbrennen. Dann sagte er, daß er von jetzt an die Totonaken für seine Brüder hielte. Sofort ließ er im Heiligtum ein Marienbild aufstellen, einen Altar konstruieren, eine Messe feiern. Die acht Mädchen, die ihm die Indios als Gastgeschenk gegeben hatten, ließ er nach einer Kurzkatechese taufen und unter die Soldaten verteilen. ${ }^{24}$

Cortés war nicht etwa ausnahmsweise grausam. Schon der erste ethnographische Bericht über die ,Conquista Espiritual' Amerikas, vom Hieronimitenmönch Ramón Pané im Jahre 1496 verfaßt, zeigt die zur Beherrschung der indianischen Bilder- und Vorstellungswelt entfesselte Gewalt. Als Indios „die Statuen der Christen auf den Boden warfen, sie mit Erde zudeckten und darüber urinierten", da eröffnete Bartolomé Kolumbus, der Bruder des Entdeckeradmirals, ,einen Prozeß gegen die Übeltäter und, als die Wahrheit herauskam, ließ er sie öffentlich verbrennen". ${ }^{25}$

Die christliche Wahrheit hat ein blasses, europäisches Gesicht. Im Bordbuch seiner ersten Reise schreibt Kolumbus begeistert über die Fügsamkeit der 
Indios: „Diese Indios sind unterwürfig und gut geeignet Befehle zu erhalten, zu arbeiten, zu säen und alles andere zu tun, was notwendig ist, Dörfer zu konstruieren und zu lernen bekleidet herumzulaufen und unsere Sitten und Bräuche zu übernehmen. ${ }^{26}$

Und Pero Vaz de Caminha, der Chronist von Pedro Alvares Cabral, dem Entdecker Brasiliens, weist in seinem Bericht über die erste Begegnung zwischen Indios und Portugiesen darauf hin, daß es in Brasilien leicht sein werde zu evangelisieren, weil die Indios, die er während der ersten Messe beobachtete, alles "genau so wie wir" machten. ${ }^{27}$

Manoel Ribeiro Rocha, Verfasser des 'Etíope Resgatado', eines „theologisch-juristischen Diskurses“ zur Sklavenfrage aus dem Jahre 1758, ist ganz explizit, wenn er seine katechetische Methode in der Sklavenhütte mit einer Papageiendressur vergleicht. Um einem Papageien das Reden beizubringen, müßten „zwei Faktoren zusammenspielen“, Veranlagung und Eifer des Papageis und die Geduld und Ausdauer des Lehrers. ${ }^{28}$

Auch die Sklavenideologie - daß Sklaverei Befreiung von physischer oder das Seelenheil betreffender Todesstrafe sein könne - wurde nicht von der lateinamerikanischen Christenheit erfunden. Das Paradigma des 'resgate' hatte im christlichen Portugal Manoel Ribeiro Rochas schon eine sich über mehrere Jahrhunderte erstreckende legitimatorische Tradition. Bereits in den offiziellen Chroniken der afrikanischen Conquista ist von einem Sklaverei oder Leibeigenschaft konstituierenden und gleichzeitig seelenrettenden Loskauf (resgate) die Rede. Bei der Schilderung, wie dem Infanten D. Henrique die ersten an der afrikanischen Küste eingefangenen Sklaven vorgestellt wurden, schrieb Gomes Eanes da Zurara im Jahre 1453 in seiner Chronik von Guiné: „Selbst wenn ihre Leiber sich irgendwie in Knechtschaft befinden sollten, ist das eine Kleinigkeit im Vergleich zu ihren Seelen, die nun auf alle Ewigkeit wahrhaftige Freiheit haben werden". ${ }^{29}$

Das religiöse Argument der Seelenrettung allein war aber nicht ausreichend, um die Wiedereinführung der Sklaverei großen Stils, seit der zweiten Hälfte des 15. Jahrhunderts, zu begründen. Das Paradigma vom 'resgate' meinte Befreiung von Leib und Seele. In der Rechtssprache der Conquista bedeutete 'resgate' daher auch Umwandlung einer härteren Strafe in eine leichtere, so zum Beispiel der Todesstrafe, welche die Afrikaner angeblich für ihre Stammesfeinde vorgesehen hatten, in lebenslängliche Zwangsarbeit und Sklaverei. Eanes da Zurara beschreibt, „wie Antão Gonçalves den ersten Loskauf bewerkstelligt hat: ihr müßt nämlich wissen“, schreibt er, „daß die Neger (...) schon Leibeigene des andern sind aufgrund alter Bräuche, die, wie ich glaube, von der Verdammung herrühren, die Noe nach der Sintflut über seinen Sohn Kam herabgerufen hatte". 30

Eine in Sklaverei oder Leibeigenschaft umgewandelte Todesstrafe bedeutet eben nicht Tod, sondern Leben. Die gängige Argumentationsfigur lautete: „besser ein lebender Sklave als ein zum Tode verurteilter afrikanische Kriegsgefangener, besser ein christlich-erlöster Sklave als ein im wörtlichen Sinne verdammt-freier Heide“. 
Diese nicht mehr legitimationspflichtige Sklaverei erlaubte es dem ersten Jesuitenprovinzial Brasiliens, Manuel da Nóbrega, seinen Mitbruder Luís da Grã als aufmüpfigen „Eiferer der heiligen Armut" zu ironisieren. Dieser verlange vom Orden, so Nóbrega in einem Brief an den Ordensgeneral, „weder landwirtschaftlichen Grundbesitz noch Sklaven" zu haben. ${ }^{31}$ Und der erste Nachfolger des Ordensgründers Ignatius von Loyola, Pater Diego Laynes, billigte der Gesellschaft Jesu in Brasilien die zum Betrieb der Kollegien und Internate notwendigen Sklaven zu, sofern diese rechtmäßig erworben worden seien. „Sklaven zu haben für den Unterhalt der Rinderfarmen, zum Fischen oder für sonstige Arbeiten, die zur Aufrechterhaltung solcher Häuser notwendig sind, halte ich nicht für unangebracht", lautete die Rückmeldung des Ordensgenerals vom 16. Dezember $1562 .{ }^{32}$ Als die Gesellschaft Jesu Kollegien und Internate als Mittel zur Katechese und Zivilisation einsetzte, entschied sie sich - wie auch andere Orden für den ordenseigenen Großgrundbesitz und für Sklavenarbeit. Daß der Sklavenbesitz, besonders für die Orden, die Gretchenfrage nach der Evangelischen Armut, nach der christlichen Brüderlichkeit und nach dem Arbeitsethos überhaupt aufwarf, wurde nur von prophetischen Außenseitern erkannt.

Aus der "ökonomischen Notwendigkeit“ wurde eine „katechetische Rechtmäßigkeit". In der lateinamerikanischen Christenheit wird Sklaverei zur legitimen Evangelisationsmethode. So pries etwa 70 Jahre später der Jesuitenprovinzial Antonio Vieira, der sich übrigens leidenschaftlich für die Freiheit der Indios einsetzte, die Sklaverei von Afrikanern in Brasilien als "großes Wunder göttlicher Vorsehung und Barmherzigkeit“. Den Sklaven einer Zuckermühle in Bahia konnte er im Jahre 1633 zurufen: „Oh, wenn doch die Schwarzen, die aus der Wildnis ihres Äthopiens herausgeholt und nach Brasilien gebracht wurden, recht erkennen würden, wie sehr sie Gottes und ihrer heiligsten Mutter Schuldner geworden sind durch das, was als Verbannung, Gefangenschaft und Unglück erscheinen könnte, aber in Wirklichkeit ein Wunder, ein großes Wunder ist! Sagt mir: eure Eltern, die in der Finsternis des Heidentums geboren sind und in ihr leben und ihr Leben beenden ohne das Licht des Glaubens und ohne Gotteskenntnis - wohin kommen sie nach dem Tode? Alle (...) kommen sie in die Hölle, und dort brennen sie jetzt und werden brennen für alle Ewigkeit“".33

Wie sollen die Nachfahren der Sklaven all jene biblischen Texte lesen, die von der Befreiung aus der Sklaverei Ägyptens sprechen, wo doch die Begegnung mit dem Christengott der Beginn der Sklaverei ihrer Väter war? Für aus Afrika kommende Sklaven stellte sich die Gewissensfrage in den Ordenshäusern nur bei „Außenseitern“. Am Ende des 16. Jahrhunderts erhielten die Jesuiten Angolas vom Stamm der Sobas jährlich neben Bodenprodukten auch 300 Sklaven, von denen sie 150 zum Unterhalt ihrer Werke verkauften. ${ }^{34}$ In einem Brief vom 21. August 1611 an seinen Mitbruder Alonso de Sandoval meinte Pater Luis Brandon, Rektor des Jesuitenkollegs in Luanda: „Wir sind hier seit 40 Jahren (...) und wir und die Patres von Brasilien kaufen diese Sklaven für unsere Arbeiten ohne Skrupel. (...) Unter zehn- oder zwölftausend Negern, die jährlich in diesem Hafen verschifft werden, einige herauszusuchen, die unrechtmäßig versklavt wurden, ist selbst unter größten Vorsichtsmaßnahmen unmöglich. Wegen einigen unrechtmäßig Versklavten, die man ja nicht kennt, all die Seelen zu verlieren, die hier verschifft werden und von denen viele sich retten, das scheint 
- da es sich ja um wenige Zweifelsfälle handelt - nicht der Wille Gottes zu sein, weil es sich bei den Geretteten und rechtmäßig Versklavten ja um viele handelt." 35

Selbst wenn es kein Christentum gäbe, wäre, angesichts der Todesstrafe, der Loskauf in die Sklaverei das kleinere Übel. Christliche Sklaverei aber fügt dem ersten Vorteil vorläufiger Lebensrettung den zweiten endgültiger Seelenrettung hinzu. Auf diese Weise konnte ein zum Loskauf der Sklaven gegründeter Orden selbst zum Sklavenbesitzer werden: Als die Mercedarier im Jahre 1794 aus der Provinz Pará vertrieben wurden, da gehörten zu ihren vom Staat eingezogenen Gütern neben großen Ländereien und Rinderfarmen mit 30.000 Ochsen, 40.000 Kühen und 8.000 Pferden auch 375 Sklaven. ${ }^{36}$

Als Kirche und religiöse Orden vergessen hatten, daß der Auftrag des Herrn lautete, Gefangene zu befreien (Lk 4,18f) und nicht Freie zu versklaven, da wurden sie selbst zu Sklavenhaltern. Im Jahre 1775 hatten die 81 Klarissen von Salvador da Bahia z. B. 290 Sklavinnen, 40 freigelassene Sklavinnen und 8 Sklaven. ${ }^{37}$ Nahezu alle Orden in Brasilien waren, bis kurz vor der offiziellen Abschaffung der Sklaverei im Jahre 1888, Sklavenbesitzer. Und viele religiöse Gemeinschaften hatten noch bis zum Konzil statutarische Beschränkungen für die Zulassung von Afroamerikanern zum Ordensstand.

Der "christliche Zeitgeist" war grundsätzlich mit der Negersklaverei einverstanden. Die Kirche sah ihre Aufgabe darin, daß Sklaven innerhalb der kolonialen Systemlogik "menschlich" und "christlich" behandelt wurden. Sie versuchte, Sklaven vor den Übergriffen ihrer Herren zu schützen und sie zu den Sekundärtugenden Frömmigkeit, Fleiß,Geduld und Gehorsam anzuleiten.

Die Freiheit der Indios war von päpstlichen Dokumenten seit dem Jahre 1537 im Abstand von jeweils 100 Jahren sehr energisch eingefordert worden. Gegen die Negersklaverei gibt es ähnliche Dokumente erst im 19. Jahrhundert. ${ }^{38}$ Ein in der sozialen Frage sehr weitsichtiger Papst wie Leo XIII. konnte noch im Jahre 1888 in seiner Enzyklika zur Abschaffung der Sklaverei behaupten, daß gegenüber der "grausamen und kriminellen“ heidnischen Sklaverei, die christliche Sklaverei voller „Süßigkeit und Humanität" gewesen sei (In Plurimis, Nr. 22). Beim Anlaß der vierhundertjährigen Kolumbusfeier sah der gleiche Leo XIII. die Ureinwohner Amerikas noch als „in bejammenswerter Finsternis sinnlosen Gebräuchen und dem Glauben an eingebildete Götzen ergeben“, während er Kolumbus das Verdienst zuschrieb, ,das Christentum und die Wohltaten der christlichen Liebe nach Westen" ausgebreitet zu haben. ${ }^{39}$

Ja selbst ein so weitgereister Zeitgenosse wie Papst Johannes Paul II. sagte noch den Teilnehmern eines Symposiums über „Geschichte der Evangelisation in Amerika" im Mai 1992, daß es sich bei der Conquista um ein "Treffen europäischer Völker mit den Völkern des amerikanischen Kontinents" gehandelt habe. Der Papst sprach "vom großartigen und bewundernswerten Abenteuer der Entdeckung und ersten Evangelisation“. Die Indios seien die „wirklichen Herren ihrer Güter gewesen“, „trotz kultureller Mängel“, trotz „fehlender Erziehung und menschlicher Bildung". Aber aufgrund des internationalen Menschen- 
rechts auf Begegnung und Kommunikation hätten „besser begabte Menschen und Völker die Pflicht gehabt, zurückgebliebene und unterentwickelte Völker zu unterstützen". ${ }^{40}$

Das Indios und versklavten Afrikanern im lateinamerikanischen Evangelisationsprozeß abverlangte Vergessen ihrer eigenen Vergangenheit kann nicht einfach durch die Sprachregelung vom „Zusammentreffen der Kulturen“ entdramatisiert werden. Viele autochtone Traditionen wurden zu einem Katakombendasein im religiösen Untergrund verurteilt. Nach der Zerstörung ihrer Tempel haben die Indiopriester auf den entlegenen Bergen und in der Nacht der Wälder ihre Geschichte weitererzählt und den 'padrecito' mit auswendig gelernten Katechismusantworten abgespeist. Die Bewohner der Sklavenhütte haben unter der Maske christlicher Heiliger afrikanische Geister und Götter gefeiert. Wer die Macht hat, kann Zeichen und Symbole weltweit kontrollieren. Die Inhalte und die damit verbundenen Vorstellungen jedoch sind unkontrollierbar. Sehr viele religiöse Manifestationen der Kolonialzeit - betrachtet man sie unter dem Prisma offizieller Erwartungshorizonte - waren Potemkinsche Dörfer.

Daher ist die Frage, ob es sich bei der Christianisierung Lateinamerikas nun um einen Religionswechsel oder um Evangelisation gehandelt habe, gar nicht so abwegig. Der vernichtete, ausgeschlossene oder zwangsintegrierte Andere signalisiert Genozid, Ghetto und Reduktion. An diesem Anderen zeigt sich Kohärenz und Ambivalenz des Christentums. Und diese Ambivalenz kann nicht einfach anderen Jahrhunderten, anderen Konfessionen, Klassen, Kulturen oder auch anderen Sektoren der eigenen Kirche angelastet werden. Sie ist eine Bedingung von Geschichtlichkeit. Wie ist es möglich, die Flurschäden diese Ambivalenz des Christentums zu begrenzen?

\section{Befreiung des Anderen}

Es wäre historisch unkorrekt, die faktische Rolle des Christentums in Lateinamerika auf die Rolle einer Unterdrückungsideologie zu reduzieren. Jeder manichäistische Erklärungsversuch der historischen Präsenz des Christentums würde gerade seine Ambivalenz übersehen und die goldenen Zierleisten „hoher Ansprüche" durch den angekohlten Rahmen einer "schwarzen Legende" ersetzen. Die Geschichte zeigt, daß es neben dem angepaßten Christentum immer auch das "andere" Christentum gegeben hat, das all jenen zur Seite stand, die durch Widerstand und Hoffnung, durch Trauer und Schweigen gegen den Stachel der Trostlosigkeit und des Todes gelöckt haben.

Der Erzbischof von Mexiko, Alonso de Montúfar, äußerte schon sehr früh Unverständnis über den gesetzgeberischen Eifer, mit dem die Spanische Krone einseitig versuchte, die Freiheit der Indios zu garantieren. Dies stünde ganz im Gegensatz zu dem, „was in diesen Gegenden mit den Negern geschähe, die in Schiffen aus allen Teilen Guineas und aus den von Portugal eroberten Ländern kämen“. Und Montúfar bringt dieFrage auf den entscheidenden Punkt, wenn er fortfährt: „Wir wissen nicht, welchen Grund es dafür geben mag, daß die Neger mehr Gefangenschaft verdienen sollen als die Indios, denn, wie ich höre, nehmen sie guten Willens das Heilige Evangelium an und führen keinen Krieg 
gegen die Christen", was gemeinhin als Grund für die Sklaverei angeführt wird. ${ }^{41}$ Eine schlüssige Antwort auf diese Frage hat er wohl nie erhalten.

Manuel da Nóbrega, der erste Jesuitenprovinzial Brasiliens, schrieb am Epiphaniefest des Jahres 1550 aus Porto Seguro: „In diesem Land haben alle oder der größte Teil der Leute ein schlechtes Gewissen wegen der versklavten Indios, die sie gegen die Vernunft besitzen $(\ldots)^{\prime}{ }^{42}$

Und sein Mitbruder Miguel Garcia schreibt im Jahre 1583, zu einer Zeit, als sein Jesuitenkolleg in Bahia 70 Sklaven hatte, daß kein Sklave Brasiliens rechtens gefangen sei. Seinem Ordensgeneral Aquaviva bekennt er: „Manchmal kam es mir in den Sinn, daß ich sicherer Gott dienen und mich retten würde in der Welt als in der Ordensprovinz, wo ich die Dinge sehe, die ich sehe. (...) Die große Zahl von Sklaven, welche die Gesellschaft in dieser Provinz und speziell in diesem Kolleg hat, ist eine Sache, die ich auf keinen Fall hinunterschlucken kann, besonders, weil nicht in meinen Kopf hinein will, daß sie rechtmäßig erworben wurden". ${ }^{43}$

Gonçalo Leite, Zeitgenosse von Miguel Garcia und ebenfalls Professor am Jesuitenkolleg in Bahia, sah durch die Kollaboration mit dem Zeitgeist sein Seelenheil in Frage gestellt: „Die nach Brasilien gehen, gehen nicht, um Seelen zu retten, sondern um ihre eigenen zu verdammen. Weiß Gott, mit welchem Schmerz ich dies schreibe, denn ich sehe unsere Patres Mördern und Räubern von Freiheit, Gut und Schweiß anderer die Beichte abnehmen, ohne Wiedergutmachung der Vergangenheit und ohne Heilmittel zukünftiger Übeltaten, die auf gleiche Weise täglich begangen werden" ${ }^{44}$ Der Bericht des Ordensvisitators Cristóvão de Gouveia, der Pater Gonçalo für „unangepaßt“ und „unruhig“ hielt, sorgte dafür, daß dieser nach Portugal "zurückgerufen“ wurde.

Neben Miguel Garcia und Gonçalo Leite wären noch andere solcher "Außenseiter" zu nennen, die sich nicht an den Mast des Zeitgeistes fesseln ließen. Es müßten die beiden Kapuzinerpatres Francisco José de Jaca und Epifanio de Moirans genannt werden. Der ein von Cartagena (Kolumbien) - wo Alonso de Sandoval und Pedro Claver wirkten -, der andere von Cumaná (Venezuela) kommend, lernten sie sich im Hafen von Havanna kennen. Als sie in Wort und Schrift für die Freilassung der Sklaven eintraten, wurde ihnen 1681 der Prozeß gemacht. Sie wurden eingesperrt und mußten nach Spanien zurückkehren. ${ }^{45}$

Schließlich soll noch - ebenfalls aus dem 17. Jahrhundert - der kühne Bericht erwähnt werden, den die Kapuzinerpatres Francisco de la Mota und Angel de Fuente la Peña, auch im Namen ihrer Mitbrüder, aus ihrer Mission an der Küste Guineas an den König von Portugal gerichtet haben. Sie nannten darin den Sklavenhandel "unerlaubt, sündhaft und ungerecht" und forderten, daß er "verboten werde und daß allen Sklaven, die in dieser Gegend gekauft wurden (...) die Freiheit zurückerstattet werde“ ${ }^{46}$

Die Spurensuche nach diesem „anderen“, von offiziellen Quellen oft boykottierten Christentum, wird in unseren historischen Seminaren noch nicht energisch genug vorangetrieben. In jedem Jahrhundert gab es Christen, die den sog. Epochengeist gegen den Strich gebürstet haben. Christentum kann eben nicht 
auf ein aseptisch-offizielles Christentum reduziert und Kirche kann nicht auf Hierarchie zurückgestutzt werden. Die Bedeutung des Christentums für die Sklavenhütte ging über die Katechismuswahrheiten des aus dem Herrenhaus kommenden Mietlings weit hinaus.

Die nachkonziliare Erneuerung durch Medellín und Puebla hat Kirche und Volk in Lateinamerika einander näher gebracht. Dafür stehen die Namen qualifizierter Zeugen: Enrique Angel Angelelli (Bischof von La Rioja, Argentinien; †04.08.1976), Luis Espinal (Jesuit, Bolivien; †22.03.1980), Henrique Pereira Neto (brasilianischer Diözesanpriester; †12.08.1974), Tito de Alencar (Dominikaner, Brasilien; †12.08.1974), Rudolf Lunkenbein (Salesianer, Brasilien; †15.07.1976), Rutilio Grande (Jesuit, El Salvador; †12.03.1977), Oscar Arnulfo Romero (Erzbischof von San Salvador; †24.03.1980) und die sechs Jesuiten der Katholischen Universität von El Salvador (†15.11.1989), Vicente Menchu (Katechet,Guatemala; †31.01.1980), 14 Märtyrer von Olanco (Honduras; U†24.06.1975), Hector Gallego (Diözesanpriester, Panama; †09.06.1981), Alvaro Ulcue (indianischer Priester aus dem Stamm der Paez; Ü† 10.11.1984), Bischof Alejandro Labaka Ugarte und Schwester Agnes Arango (Aguarico, Equador; $\dagger 21.07 .1987$ ).

Diese Brückenbauer zwischen Volk und Kirche, diese Grenzgänger zwischen Leben und Tod, diese Zeugen der großen Drangsal haben neue Perspektiven kirchlicher Präsenz inmitten derer eröffnet, die aus dem Dunkel der „verlorenen" Jahrhunderte kommen. An der Seite dieser Opfer beginnen Sektoren in den verschiedensten Kirchen zu begreifen,

- daß die Anderen von morgen nicht nur befreite Arme von gestern sein werden;

- daß die Anderen nicht nur das Spiegelbild der Gleichen sein wollen, so wie die Frauen auch nicht darum kämpfen, „andere Männer" zu werden;

- daß die Zukunft der Anderen nicht die Gegenwart der heute Herrschenden sein wird;

- daß die Sache der Anderen nicht mit Mestizentum, Akkulturation, Synkretismus, Rassenlaboratorium oder Androgynie abzugelten ist;

- daß identitätsstiftendes Anderssein nicht legitimationspflichtig ist.

So enthält die Sache der Anderen einen Stachel, der gegen Neokolonialismus, politische Hegemonie, solipsistische Wahrheitsansprüche und graue Uniformität gerichtet ist. Die andere Kirche als Kirche der Anderen wird eine pfingstlich-vielfältige, dezentralisierte und auf einen universalen Befreiungshorizont hin artikulierte, dialogische Ökumene sein. Sie wird nicht mehr nur advokatorisch Stimme der Wortlosen, sondern sie wird Stimme der Anderen sein. Als Stimme der Anderen ist sie kritisch, denunziert den ideologischen Umgang mit Geschichte und klagt gesamtmenschheitliche Solidarität und Toleranz ein. Die Stimme der Anderen ist jene prophetische Stimme, die das Einspruchsrecht all derer, denen vorzeitig das Leben durch Genozid, Ghetto oder Gleichmacherei genommen wurde, zur Sprache bringt. Sie ist die Stimme der anderen Zukunft, deren Möglichkeit sie durch die Einspruchspflicht historischer Quellen und dialogischer Vernunft - gegen die Zwänge der Widerholung, des Schicksal und der Sachen - stets neu erinnert. 
Anmerkungen:

1 Cristobal Colon, Textos y documentos completos, Madrid (Alianza, AU 320) 1984, 15. Vgl. Christoph Kolumbus, Bordbuch, Frankfurt (Insel, it 476) 1981.

2 Vgl. Angel Losada, Fray Bartolomé de Las Casas a la luz de la moderna crítica histórica, Madrid (Ed. Tecnos) 1970, 33 f.

3 Bartolomé de Las Casas. Historia de las Indias, Buch 1, Kap. 155.

4 Colección de documentos inéditos relativos al descubrimiento, conquista y organización de las antiguas posesiones españolas de América y Oceanía, sacados de los Archivos del Reino y mui especialmente del de Indias (D.I.I.), Madrid 1864-1889, Bd. $31,13$.

5 Ebd., Bd.31, 209.

6 Las Casas, Historia, 1.c., Buch 1, Kap. 160. Vgl. auch Buch 2, Kap. 1.

7 Vgl. José Maria Chacon y Calvo, Cedulario cubano, los origens de la colonización (1493-1512), Madrid (Compañia Ibero-Americana de Publicaciones, Colección de Documentos Inéditos para la Historia de America, Bd. 6) 1929, 129.

8 Vgl. zur Konzessionsbulle 'Universalis Ecclesiae' Pedro de Leturia, Der Heilige Stuhl und das spanische Patronat in Amerika, in: Historisches Jahrbuch 46 (1929) $2 \mathrm{ff}$.

$9 \mathrm{Vgl}$. Theodorus Mommsen, Monumenta Germaniae Historica. Chronica minora. Saec. IV., V., VI., VII. Bd. II., Berlin (Ed. Weidmann) 1894, 352ff.

10 Bartolomé de Las Casas, Historia de las Indias, Buch 3, Kap. 138.

11 Memorial de [14] remedios para las Indias, in: Obras escogidas de Fray Bartolomé de Las Casas. Opusculos, cartas y memoriales. Bd. 5 BAE (110), Madrid 1958, 5-27; hier: 16b, 17a. Vgl. Historia de las Indias, Buch 3, Kap. 85, 102/129.

12 Vgl. Memorial de [5] Remedios para las Indias, de 1518, in: Obras escogidas de Fray Bartolomé de Las Casas. Opusculos, cartas y memoriales. Bd. 5 BAE (110), Madrid 1958, 31-35, hier: 34a/b.

Memorial de Remedios [1518], Barcelona, ebd., 35-39, hier: 35a, 39a/b.

Proyecto de capitulacion y asiento con el emperador Carlos V para la pacification y conversion de la tierra firme, Barcelona 1519, in: Historia de las Indias, Buch 3, Kap. $132 \mathrm{BAE}, 96,493 \mathrm{~b}$.

Carta al consejo de Indias [20.01.1531], in: Obras escogidas, 1.c. Bd. 5 BAE (110), 4355, hier: $54 \mathrm{~b}, 55 \mathrm{a}$.

Memorial de remedios, de 1518, ebd., Bd. 5 BAE (110), Madrid 1958, 120-123, hier: 121a.

13 Peticion al emperador Carlos V, Suplica 22, in: Helen Rand Parish, Las Casas, opispoLas Casas as a Bishop, Washington (Library of Congress) 1980, 9.

14 Vgl. Antonio Maria Fabie, Vida e escritos de fray Bartolomé de Las Casas, opispo de Chiapa. Bd. 2, Madrid 1879, 96.

$15 \mathrm{Vgl}$. Bartolomé de Las Casas, Historia de las Indias. Buch 3, Kap. 102 und 129.

16 Vgl. Bernhard Blumenkranz, Die Judenpredigt Augustins, Basel 1946; ders., Les auteurs chrétiens latins du Moyen Age sur les juifs et le judaísme, Paris 1963; Jeremy Cohen, The friars and the jews. The evolution of medieval anti-judaism, Ithaca/ London 1982; Lidia Storoni Mazzolani, Sant' Agostino e i pagani, Palermo (Sellerio Editore) 1987.

17 Ambrosius, Epist. 40 PL 16, 1104 ss.

18 San Bernardo, Obras Completas, Bd. 1, Madrid (B.A.C.) 1983, 503

19 Vgl. José Luis Martin, Origenes de la orden militar de Santiago (1170-1195), Barcelona (CSIC) 1974

20 Bullarum Diplomatum, Bd. 5, 11-115.

21 Fernando Cortés, Cartas y documentos, Mexiko (Porrúa, BP 2) 1963, 50.

22 Emilio Choy, De Santiago matamoros a santiago mata-indios, in: Antropologia e historia 1 (1979) (Lima, Ed. Universidad Nacional Mayor de San Marcos) 333-437.

23 Bernal Diaz del Castillo, Historia verdadera de la conquista de la Nueva España. 2 Bde., Mexiko (Porrúa, BP 6/7) 1980, hier: Bd. 1, 161 (Kap. 51).

24 Vgl. ebd., 162f. (Kap. 52).

25 Ramón Pane, Relación acerca de las antigüedades de los indios, Mexiko (Siglo Veinti- 
uno) 81988 , 46f. (Kap. 26).

26 Cristóvão Colombo, Diários da descoberta da América, Porto Alegre (L\&PM) 1984, 74.

27 Silvio Castro (Hg.), A carta de Pero Vaz de Caminha. O descobrimento do Brasil, Porto Alegre (L\&PM) 1985, 94f.

28 Manoel Ribeiro Rocha, Etiope resgatado, Petrópolis (Ed. Vozes) 1992, 110 (VI,10).

29 Gomes Eanes da Zurara, Crónica de Guiné (1453), 80.

30 Ebd., 85.

31 Brief des brasilianischen Provinzials Manuel da Nóbrega an den Ordensgeneral Diego Laynes vom 12.06.1561 (S. Vicente), in: SerafimLeite (Hg.), Cartas dos primeiros Jesuítas do Brasil. Bd. 3, São Paulo 1954, 354-366, hier: 364f.

32 Ebd., 514

33 Antônio Vieira, Sermão décimo quatro (1633), in: Sermòes. Bd. 4, Teil 11, Nr. 6, 301

34 Louis Jadin, L'oeuvre missionnaire en Afrique noire, in: J. Metzler, Sacrae congregationis, Bd. I/2, 441f.

35 Alonso de Sandoval, Un tratado sobre la esclavitud, Buch 1, Kap. 17, $143 f$.

36 Vgl. Ernesto Cruz, Mercedários no Pará, in: Revista de Cultura do Pará, II/6-7 (Jan.Juni 1972) 97-104, hier: 103f. Der Orden sei, so die Begründung für den Besitz von Sklaven, zum Loskauf von Christen aus muslimischer Gefangenschaft gegründet worden, deren Glaube in Gefahr gewesen sei. Für von Christen versklavte Afrikaner habe diese Gefahr ja nicht bestanden.

37 Vgl. Riolando Azzi/Maria Valéria V. Rezende, A vida religiosa feminina no Brasil Colonial, in: Riolando Azzi (Hg.), A vida religiosa no Brasil, São Paulo (Cehila/Ed. Paulinas) $1983,50$.

38 Sublimis Deus (1537) v. Paul III., Commissum Nobis (1639) v. Urban VIII. und Immensa pastorum (1741) v. Benedikt XIV. an die Bischöfe Brasiliens und Portugals gerichtet, treten für die Freiheit der Indios ein. Gegen die Negersklaverei nehmen der Apostolische Brief In supremo (1839) v. Gregor XVI und der an die brasilianischen Bischöfe gerichtete Brief In Plurimis (1888)v. Leo XIII. Stellung.

39 Unseres Heiligen Vaters Leo XIII. Schreiben an die Erzbischöfe und Bischöfe von Spanien, Italien und Nord- und Südamerika über Christoph Columbus (16.07.1892), in: Die katholischen Missionen, XX/10 (Akt. 1892) 201-203, hier: 202.

40 Johannes Paul II., Nova estratégia evangelizadora para o continente latino-americano, in: L'Osservatore romano, 21 (24.05.1992) 5 (portugiesische Ausgabe). Das Symposium wurde von der päpstlichen Kommission für Lateinamerika vom 10.14.05.1992 im Vatikan durchgeführt.

41 Brief des Erzbischofs Montúfar an den König vom 30.06.1560, in: Francisco del Paso y Troncoso, Epistolario de Nueva España. Bd. 9 (1560-1563), Mexiko 1940, 53f.

42 Cartas Jesuíticas I: Manoel da Nóbrega, Cartas do Brasil (1549-1560), Bela Horizonte/ Sào Paulo 1988, 109.

43 Der Brief ist vom 26.01.1583 (Bahia), in: Archivum Societatis Iesu Romanum (ASIR), Lusitania, 68, 255.

44 Brief des Paters Gonçalo Leite an den Ordensgeneral vom 20.06.1586, Lissabon, in: ASIR, Lusitania, 69, 243.

45 Vgl. José Tomás Lopez Garcia, Dos defensores de los esclavos negros en el siglo XVII, Maracaibo/Caracas 1981.

46 Informe y relacion que Fr. Francisco de la Mota (...) [14.04.1684], in: As viagens do bispo D. Frei Vitoriano Portuense a Guiné e a cristanizaçào dos reis de Bissau. Junta de Investigaçòes Cientificas do Ultramar, Lissabon 1974, 121-133, hier: 121. 


\section{SUMMARY}

Against the background of the current discussion about the "Quinto Centenario" of the 'discovery' and conquest of Latin America the author opposes the frequent reduction of the 'other' to the 'poor'. Beside the necessary socio-analytical perspective one needs above all an ethnic-cultural perspective which takes serious the cultural variety and otherness of many latin american population groups. The basic question of the latin American ecclesiastical history runs: Has there actually been an 'evangelization' or only a 'christianization'? The author turns out that as far as the church is concerned a merciful acceptance of the poor has often gone hand in hand with a confused intolerance with regard to the other. After the Conquista many old-established traditions of latin american people were condemned to a catacomb life in the religious underground. After the destruction of their temples the Indian priests have quite frequently gone on telling their story on removed hills and in the darkness of the forests, fobbing the catholic priests off with answers from the catechism learned by heart. Yet, despite some forms of resistance the exterminated, isolated or compulsively 'integrated' other demonstrates the violence and the potential of suppression of the christianization of Latin America.

History proves, however, that beside the official Christianity in Latin America there has always been the 'other' Christianity which defends the cause of the others and stands up against neo-colonialism, political hegemony, eurocentric claims for truth and grey uniformity.

\section{RÉSUMÉ}

Dans la discussion actuelle sur le "Quinto Centenario" de la "découverte" et de la conquête de L'Amerique Latine l'auteur s'oppose à ce qu'on réduise souvent "l'autre" en „pauvre". Outre la perspective socio-anaytique nécessaire il faut surtout une perspective ethno-culturelle qui prenne au sérieux la diversité et la différence de beaucoup de groupes ethniques américo-latines. La question fondamentale de l'histoire de l'église en Amérique Latine est: Y a-t-il en une veritable "évangelisation“ ou seulement une "christianisation"?

A l'eglise on s'aperçoit qu'il existait souvent une acceptation miséricordieuse du pauvre à côte d'une intolérance irritante vis-à-vis de l'autre. Après la conquête, beaucoup de traditions autochtones de peubles latino-américaines furent condamnées à vivre dans le sous-sol religieux. Après la destruction de leurs temples les prêtres indiens continuèrent souvent à raconter leur histoire sur des montagnes lointaines et dans la nuit des forêts. Et il repurent les prêtres catholiques de réponses catéchistiques qu'ils avaient appris par coeur. Malgré certaines formes de résistance, l'autre qui est détruit, exclu ou „intégré“ par coutrainte prouvre la brutalité et la puissance répressive de la christianisation de l'Amerique Latine.

Cependant l'histoire montre qu'à côté du christianisme officiel il a toujours existé "l'autre" christianisme qu défend la cause des autres et qui lutte contre le néocolonialisme, l'hégémonie politique, les prétentions eurocentriques à la vérité et l'uniformité grise. 


\section{RESUMEN}

Teniendo como trasfondo la actual discusión en torno al "Quinto Centenario" del "descubrimiento" y conquista de América Latina, el autor se vuelve contra la frecuente reducción del "otro“ a „el pobre“. Junto a la necesaria perspectiva socialanalítica, es necesario, sobre todo, una perspectiva étnico-cultural que tome en serio la variedad cultural y la "otridad“ (el ser diferente, otro) de muchos pueblos latinoamericanos. La preguenta fundamental de la historia de la iglesia latinoamericana es: Se produjo realmente una „evangelización" o solamente una "cristianización“. Se muestra que en la Iglesia, una misericordiosa aceptación del pobre va acompañada, a menudo, de una irritada intolerancia frente al que es otro. Muchas tradiciones autóctonas de los pueblos latinoamericanos fueron condenados, después de la conquista, a una existencia de catacumbas en una clandestinidad religiosa. Después de la destrucción de sus templos, no pocos sacerdotes indígenas continuaron contando sus historias en las alejadas montañas y en la noche de las selvas y los bosques, despachando a los sacerdotes católicos con respuestas de catecismo aprendidas de memoria. Pero a pesar de algunas formas de resistencia, el Otro, destruído, excluído o „integrado“ a la fuerza muestra la violencia y el potencial de opresión de la cristianización de América Latina.

Pero la historia muestra que junto al cristianismo oficial, en América latina se ha dado tambien el "otro" cristianismo que defiende la cuestión del Otro y que lucha contra el neocolonialismo, la hegemonía política, las pretenciones eurocéntricas de posesión de la verdad y contra la gris uniformidad. 